EPJ Web of Conferences 107, 07002 (2016)

DOI: $10.1051 /$ epjconf/201610707002

(C) Owned by the authors, published by EDP Sciences, 2016

\title{
Collective aspects of microscopic mean-field evolution along the fission path
}

\author{
Yusuke Tanimura $^{1, a}$, Denis Lacroix ${ }^{1, b}$, and Guillaume Scamps ${ }^{2, c}$ \\ ${ }^{1}$ Institut de Physique Nucléaire, IN2P3-CNRS, Université Paris-Sud, F-91406 Orsay Cedex, France \\ ${ }^{2}$ Department of Physics, Tohoku University, Sendai 980-8578, Japan
}

\begin{abstract}
We propose a new method to extract the collective masses and momenta associated with a given set of collective coordinates, along a dynamical microscopic mean-field evolution. We apply our method to the symmetric fission of ${ }^{258} \mathrm{Fm}$ nucleus, and analyze the dynamical evolution of the system in the collective space. We compare, between the dynamical and the adiabatic paths, the force acting on the quadrupole degree of freedom, which is closely related to the relative distance between fragments. It is shown that dynamical effects beyond the adiabatic limit are important for formation and scission of the neck between emitted fragments.
\end{abstract}

\section{Introduction}

Nuclear time-dependent energy density functional (TDEDF) approach is experiencing nowadays a renewal of interest [1-12]. It allows one to describe a wide variety of dynamical processes ranging from small to large amplitude collective motion, including nuclear reactions. Among them, the description of the nuclear fission process remains one of the most difficult challenges [13-16]. Since the process involves complex multi-dimensional quantum tunneling and dynamical effects beyond the adiabatic limit, it has not been fully described with a unified microscopic approach while the process is rather well understood based on phenomenological models [17].

The main difficulty of the fission is that it is needed to treat both single-particle and collective degrees of freedom (DOF) as quantum objects [18]. To this end, using the fact that the time scale of the fission is rather large, one can start with the adiabatic potential-energy landscape. The semiclassical approach can then be employed to obtain the fission path and lifetime based on it. Alternatively one can perform the time-dependent generator-coordinate method (TDGCM) [19] for better taking into account the manybody effect and configuration mixing. However, it is a numerically highly demanding task to perform TDGCM with several collective degrees of freedom.

As an alternative approach one can employ the timedependent energy density functional (TD-EDF) theory, which offers the possibility of describing dynamical processes involving rather complex shapes without assuming adiabaticity. A main drawback of this approach in describing the fission process is that the motion of collective DOFs is nearly classical, which means that the theory can hardly describe the quantum tunneling of the system

\footnotetext{
ae-mail: tanimura@ipno.in2p3.fr

be-mail: lacroix@ipno.in2p3.fr

ce-mail: scamps@nucl.phys.tohoku.ac.jp
}

through the fission barrier. However, it is still possible to investigate and extract some important information along the fission path after the system has passed through the barrier [13-16].

The aim of the present work is to explore the possibility to get the macroscopic transport coefficients such as mass, potential, and dissipation directly from a TD-EDF theory, which takes into account the dynamical effects that may play an important role in the fission process. To this end, we propose a new method to obtain collective masses and momenta associated with a given set of collective DOF, and we apply this method to the fission of ${ }^{258} \mathrm{Fm}$ nucleus.

\section{Collective mass and momentum extracted from dynamical mean-field theory}

\subsection{TD-EDF evolution of normal and anomalous densities}

Before starting the main subject of the present work, we recall some aspects of TD-EDF with pairing. The matrix elements of the normal and anomalous densities are defined through

$$
\rho_{i j}(t)=\left\langle\hat{a}_{j}^{\dagger} \hat{a}_{i}\right\rangle, \quad \kappa_{i j}(t)=\left\langle\hat{a}_{j} \hat{a}_{i}\right\rangle,
$$

where $\left(\hat{a}_{i}^{\dagger}, \hat{a}_{i}\right)$ correspond to creation/annihilation operators of a complete set of single-particle states. The expectation values are taken on the trial quasi-particle vacuum that is evolved in time. The TD-EDF equation of motion with pairing can be written as (see for instance [20]):

$$
\begin{aligned}
i \hbar \frac{d}{d t} \rho & =[h(\rho), \rho]+\kappa \Delta^{*}-\Delta \kappa^{*}, \\
i \hbar \frac{d}{d t} \kappa & =h(\rho) \kappa+\kappa h^{*}(\rho)-\rho \Delta-\Delta \rho^{*}+\Delta .
\end{aligned}
$$


Here $h(\rho)$ and $\Delta$ are respectively the mean-field and pairing field matrix. These operators can be generically written as:

$$
\begin{aligned}
h(\rho)_{i j} & =\left(\frac{p^{2}}{2 m_{N}}\right)_{i j}+U_{i j}(\rho) \\
& =\left(\frac{p^{2}}{2 m_{N}}\right)_{i j}+\sum_{k l} v_{i k j l}^{M} \rho_{l k} \\
\Delta_{i j} & =\frac{1}{2} \sum_{k l} v_{i j k l}^{P} \kappa_{k l} .
\end{aligned}
$$

where $m_{N}$ denotes the nucleon mass and $U$ is the meanfield potential. Here $v^{M}$ and $v^{P}$ denote effective vertex respectively in the particle-hole and particle-particle channels and can be directly defined as functional derivative of the energy.

\subsection{Mass and momentum associated with collective coordinate}

Now we give the formulas for mass and momentum of a collective variable based on the dynamical mean-field evolutions given in Eqs. (1) and (2). Here we assume that the effective interactions both in particle-hole and particleparticle channels are local and zero-range, as the ones most widely employed in the nuclear mean-field calculations. We further restrict ourselves to one-body local operators $\hat{Q}_{\alpha}$ for the collective variables. For example, it can be the quadrupole moment which is given by $\hat{Q}_{\alpha}=2 \hat{z}^{2}-\hat{x}^{2}-\hat{y}^{2}$. Its expectation value along the TD-EDF path is given by

$$
\left\langle\hat{Q}_{\alpha}\right\rangle=\operatorname{Tr}\left[Q_{\alpha} \rho(t)\right] .
$$

Let us denote the momentum conjugate to $\hat{Q}_{\alpha}$ by $\hat{P}_{\alpha}$, and the mass by $M_{\alpha}$. We demand two conditions to be satisfied by the coordinate, momentum, and mass [21], i.e.,

$$
\begin{gathered}
\frac{d\left\langle\hat{Q}_{\alpha}\right\rangle}{d t}=\frac{\left\langle\hat{P}_{\alpha}\right\rangle}{M_{\alpha}}, \\
\left\langle\left[\hat{Q}_{\alpha}, \hat{P}_{\alpha}\right]\right\rangle=i \hbar,
\end{gathered}
$$

where the latter is also used in the time-dependent randomphase approximation (TDRPA) [22]. From the above requirements we obtain [21]

$$
\begin{aligned}
& \frac{m_{N}}{M_{\alpha}}=\left\langle\left(\nabla Q_{\alpha}\right) \cdot\left(\nabla Q_{\alpha}\right)\right\rangle, \\
& P_{\alpha}=\frac{\hbar}{2 i} \frac{M_{\alpha}}{m_{N}}\left[\left(\nabla Q_{\alpha}\right) \cdot \nabla+\nabla \cdot\left(\nabla Q_{\alpha}\right)\right],
\end{aligned}
$$

for the mass and the matrix elements of the conjugate momentum in the real space, for the given collective coordinate. Similar expressions for momentum [23] and for mass (see for instance [24-26]) are also found in literature.

\subsection{Generalization to several collective degrees of freedom.}

Let us now consider a more general case where a set of $N$ collective DOFs $\left\{Q_{\alpha}\right\}_{\alpha=1, N}$ are selected. A naive generalization is to assign to each variable $Q_{\alpha}$, a collective momentum $P_{\alpha}$ with matrix elements given by Eq. (9). One should a priori also generalize the mass matrix elements as

$$
\frac{m_{N}}{M_{\alpha \beta}(t)}=\left\langle\left(\nabla Q_{\alpha}\right) \cdot\left(\nabla Q_{\beta}\right)\right\rangle .
$$

This expression naturally extends the previous case and was also given in Ref. [24]. As shown in Ref. [21], the diagonalization of the mass gives new canonical pairs of operators $\left(\hat{Q}_{k}^{\prime}, \hat{P}_{k}^{\prime}\right)$, whose commutation rules identify with the TDRPA ones and are given by

$$
\left\langle\left[\hat{Q}_{k}^{\prime}, \hat{P}_{l}^{\prime}\right]\right\rangle=i \hbar \delta_{k l} .
$$

These new operators are particularly useful to get a simple expression for the collective kinetic energy. In particular, we have $\frac{d\left\langle\hat{Q}_{k}^{\prime}\right\rangle}{d t}=\frac{\left\langle\hat{P}_{k}^{\prime}\right\rangle}{M_{k}^{\prime}}$ while the collective kinetic energy is simply given by

$$
E_{\mathrm{kin}}^{\{\alpha\}}=\sum_{k} \frac{p_{k}^{\prime 2}(t)}{2 M_{k}^{\prime}(t)} .
$$

Once the set of collective variables is properly defined, macroscopic analysis of TD-EDF evolution can be made. Such a connection from the microscopic level to the macroscopic one is illustrated below for the fission process.

\section{Application to the fission of ${ }^{258} \mathrm{Fm}$}

To illustrate the method presented in the previous section, we consider the case of ${ }^{258} \mathrm{Fm}$ that was the subject of the recent work [16]. This nucleus is anticipated to have three different paths towards fission. In this work, we concentrate on the so-called symmetric compact shape. The energy landscape is obtained using the EV8 program with a constraint of the quadrupole moment [27]. We use here the standard definition for multipole moments

$$
Q_{\lambda}=\sqrt{\frac{16 \pi}{2 \lambda+1}}\left\langle\hat{r}^{\lambda} \hat{Y}_{\lambda 0}\right\rangle
$$

leading for instance to $Q_{2}=\left\langle 2 \hat{z}^{2}-\hat{x}^{2}-\hat{y}^{2}\right\rangle$.

An illustration of the potential energy curve (PEC) is shown in Fig. 1 as a function of the quadrupole moment $Q_{2}$. As in Ref. [16], the Sly4d Skyrme functional [1] is used for the mean-field channel while a constant interaction is retained for the pairing channel. The static calculations are performed with a mesh size $13.2 \times 24.4 \times 13.2$ $\mathrm{fm}^{3}$ and a mesh step $\Delta x=0.8 \mathrm{fm}$.

The dynamical evolution of the system starting from any point of the PEC can be made consistently using the recently developed TD-EDF code including pairing in the BCS approximation [12, 28, 29]. Dynamical calculations shown here are performed in a mesh of size 


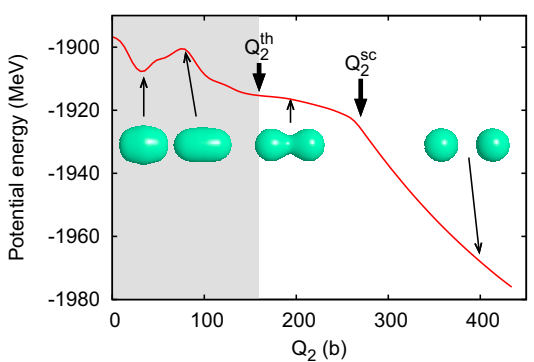

Figure 1. (Color online) Potential energy curve of ${ }^{258} \mathrm{Fm}$ nucleus as a function of the quadrupole deformation parameter (in barn unit). Isosurfaces of the total density drawn at half the maximum value at $Q_{2}=34 \mathrm{~b}, 80 \mathrm{~b}, 194 \mathrm{~b}$, and $399 \mathrm{~b}$ are also shown. The two thick arrows indicate the spontaneous fission threshold $Q_{2}^{\text {th }}$ and the adiabatic scission point $Q_{2}^{\text {sc }}$.

$26.4 \times 72.8 \times 26.4 \mathrm{fm}^{3}$ with the same mesh step as in the static case $\Delta x=0.8 \mathrm{fm}$. The time step is taken to be $\Delta t=1.5 \times 10^{-24} \mathrm{sec}$. In the present calculations, reflection and axial symmetries are assumed in the constrained calculation. Since symmetry cannot be broken spontaneously by mean-field, only even multipole moments can be nonzero during the evolution. In particular, we do not consider here possible octupole deformation.

As it was observed previously, the system will spontaneously separate into two fragments only above a certain value of the initial quadrupole moment, which is larger than that of the fission barrier shown in Fig. 1 [13-16]. The lowest initial quadrupole moment leading to spontaneous fission within TD-EDF is called hereafter "Dynamical Fission Threshold" and will be denoted by $Q_{2}^{\text {th }}$. In the present calculation, the threshold deformation is approximately $Q_{2}^{\text {th }} \simeq 160 \mathrm{~b}$. The shaded area in this figure indicates the region where the system does not spontaneously fission. The fact that $Q_{2}^{\text {th }}$ is well beyond the expected barrier position signs the deviation from the adiabatic limit of the microscopic transport theory close to single-particle level crossings (See Refs. [13-16, 21]).

\subsection{Mass parameter from TD-EDF}

In the present section, we consider different initial quadrupole deformations between the fission barrier and the scission point. The scission point corresponding to a quadrupole deformation $Q_{2}^{\text {sc }}$ has already been shown in Fig. 1. It corresponds to the kink in the PEC appearing at $Q_{2} \simeq 270$ b. Beyond the scission point, the PEC is nearly dominated by the Coulomb repulsion between the two fragments (see also Fig. 5).

As an illustration, we consider that the initial state corresponds to $Q_{2}^{\text {ini }}=160 \mathrm{~b}$, that is a situation just above the spontaneous fission threshold. In particular, it has been shown in Ref. [16] that if the system is left initially with zero collective energy, the total final kinetic energy of fragments after TD-EDF evolution is compatible with experimental observation. To study possible non-adiabatic effects, initial conditions with boosts in the quadrupole mo-



Figure 2. (Color online) Quadrupole mass parameter calculated from TD-EDF paths. In all cases, the initial quadrupole moment is $Q_{2}^{\text {ini }}=160$ b. Different trajectories correspond to different initial boosts. The corresponding initial collective energies $E_{2}^{\mathrm{ini}}$ are systematically reported in the figure. The quadrupole mass along the adiabatic PEC is also shown (solid line) for comparison.

ment of varying intensity (including no boost at all) are used. The boost in the quadrupole momentum is applied with the local operator $\exp \left(i p_{2} Q_{2}(\boldsymbol{r}) / \hbar\right)$ to each singleparticle wave-function. This induces an additional initial collective kinetic energy [14] :

$$
E_{2}^{\mathrm{ini}}=\frac{p_{2}^{2}}{2 m} \int\left|\nabla Q_{2}(\boldsymbol{r})\right|^{2} n(\boldsymbol{r}, t=0) d^{3} r
$$

where $n(\boldsymbol{r}, t=0)$ denotes the local density of the system in the adiabatic curve selected at a given initial moment.

In Fig. 2, the quadrupole mass deduced with the present method is shown as a function of $Q_{2}$ along the TD-EDF trajectories for $Q_{2}^{\text {ini }}=160 \mathrm{~b}$ with increasing initial quadrupole boosts. For display purpose, we take the ratio of the mass to its asymptotic value with two identical fragments at infinite separation. To illustrate the departure from the adiabatic path, we also show the result obtained by assuming that the local density identifies with the density obtained from the constrained mean-field calculation. In the following, the latter is referred to as "static mass".

We observe in Fig. 2 that the mass is in general rather close to the static mass, especially if $E_{2}^{\text {ini }}=0 \mathrm{MeV}$. In that case, the system first follows closely the adiabatic case and then some deviation is observed. The deviation occurs around the scission point. At this point, the slope of the PEC suddenly changes to match with the Coulomb case that dominates at large distance. This increase of slope is expected to induce also a larger collective velocity and therefore also induce a possible departure from the adiabatic limit. We see in this figure that the mass also depends on the initial collective velocity imposed to the system. The larger is the initial velocity, the more deviation from the static mass is observed.

\subsection{Total versus collective kinetic energy}

As we mentioned in Sec. 2, the present method allows us to access a set of conjugate momenta and the collective kinetic energy (CKE) as well as the masses associated with the set of collective coordinates. The CKE of the set of 


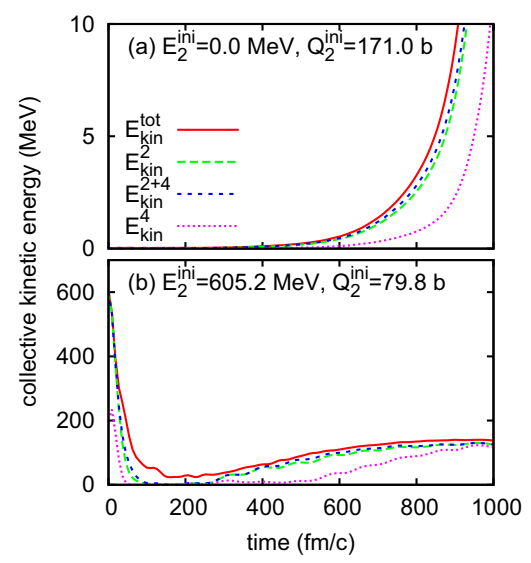

Figure 3. (Color online) Evolutions of the total collective kinetic energy $E_{\text {kin }}^{\text {tot }}$ as a function of time. The initial systems correspond respectively to a quadrupole moment (a) $Q_{2}^{\text {ini }}=171 \mathrm{~b}$ without boost or (b) to $Q_{2}^{\text {ini }}=80 \mathrm{~b}$ with initial boost. In both cases, the CKE obtained using Eq. (12) and associated to $Q_{2}$ only $\left(E_{\text {kin }}^{2}\right)$, $Q_{4}$ only $\left(E_{\text {kin }}^{4}\right)$ or both $\left(E_{\text {kin }}^{2+4}\right)$ are also shown.

collective variables $\left\{Q_{\alpha}\right\}$ can be obtained by diagonalizing the mass matrix and by using Eq. (12).

In Fig. 3, the CKE associated to the quadrupole and/or hexadecapole moments are displayed as a function of time during the fission process. We also compare these energies to the total kinetic energy computed through

$$
E_{\mathrm{kin}}^{\mathrm{tot}}=\frac{\hbar^{2}}{2 m} \int d^{3} r \frac{\boldsymbol{j}(\boldsymbol{r}, t)^{2}}{n(\boldsymbol{r}, t)},
$$

where $\boldsymbol{j}(\boldsymbol{r}, t)$ is the single-particle current. Two different initial conditions are considered, one starting from an already elongated shape without boost and one with a more compact shape but where a boost in quadrupole momentum is applied to induce fission.

From this figure several interesting aspects could be seen

- At initial time $E_{\text {kin }}^{\text {tot }}=E_{\text {kin }}^{2}$. This is indeed due to the fact that either the two are equal to zero (Fig. 3(a)) or that the initial condition (Fig. 3(b)) is such that all initial kinetic energy is induced by the quadrupole boost.

- The CKE associated to $Q_{4}$ is also initially non zero. This stems from the fact that $Q_{2}$ and $Q_{4}$ are not independent collective variables. Therefore boosting in quadrupole moment also induces an excitation of the hexadecapole and higher order even multipole moments.

- Due to the rather strong correlations between $Q_{2}$ and $Q_{4}$, the off diagonal matrix elements of the inertia play an important role. Indeed, neglecting this contribution would give:

$$
E_{\mathrm{kin}}^{2+4} \simeq E_{\mathrm{kin}}^{2}+E_{\mathrm{kin}}^{4} .
$$

However, summing directly these two energies would exceed the total kinetic energy that is an upper bound whatever is the selected set of collective variables. In

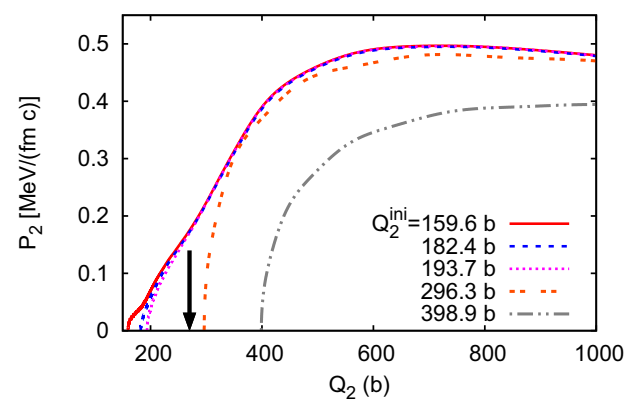

Figure 4. (Color online) Evolutions of the collective momentum as a function of time for different initial quadrupole deformations (with $Q_{2}^{\text {ini }} \geq Q_{2}^{\text {th }}$ ). The arrow indicates the scission point associated to the adiabatic potential.

Fig. 3, $E_{\text {kin }}^{2+4}$ accounts for the off-diagonal inertia and finally leads to an energy that is lower than $E_{\text {kin }}^{\text {tot }}$

- At large distances, we see that

$$
E_{\mathrm{kin}}^{\mathrm{tot}} \simeq E_{\mathrm{kin}}^{2+4} \simeq E_{\mathrm{kin}}^{2}
$$

This is due to the fact that all kinetic energies are dominated by the relative motion of the two fragments in the exit channel.

\subsection{Collective evolution close to scission}

Here we investigate the collective evolution close to the scission point. The scission can be seen directly in Fig. 1 by the change of slope around $Q_{2}^{\text {sc }} \simeq 270 \mathrm{~b}$.

The evolution of the collective momentum is displayed in Fig. 4 as a function of $Q_{2}$ for different initial deformations. We clearly see different behaviors depending if the initial quadrupole moment is above or below $Q_{2}^{\text {sc }}$. For $Q_{2}^{\text {ini }} \geq Q_{2}^{\text {sc }}$ the momentum evolution corresponds essentially to the motion of two fragments boosted by their mutual Coulomb field. For $Q_{2}^{\text {ini }} \leq Q_{2}^{\text {sc }}$, the nuclear interaction between nuclei still plays a significant role and a richer evolution is seen. In that case, independently of the initial $Q_{2}^{\text {ini }}$ value, after some transition time, all curves become nearly identical with one another.

In the absence of dissipation and assuming that the dynamics stem uniquely from a collective potential, one would expect that the smaller is $Q_{2}^{\mathrm{ini}}$, the higher is $P_{2}(t)$ as a function of $Q_{2}$. However, it is clearly seen from Fig. 4 that part of the energy is dissipated in the early stage of the evolution. To further progress, we may follow Ref. [6] and assume that the momentum evolution can be written as a simple dissipative equation of motion:

$$
\dot{P}_{2}=-\frac{\partial V_{\text {coll }}}{\partial Q_{2}}+\frac{1}{2} \frac{\partial M_{2}}{\partial Q_{2}} \dot{Q}_{2}^{2}-\gamma\left(Q_{2}\right) \dot{Q}_{2}
$$

where the collective potential $V_{\text {coll }}$ and the friction coefficient $\gamma$ are unknown quantities. In the adiabatic limit, the collective potential identifies with the one shown in Fig. 1 and $\gamma\left(Q_{2}\right)=0$ along the path. 


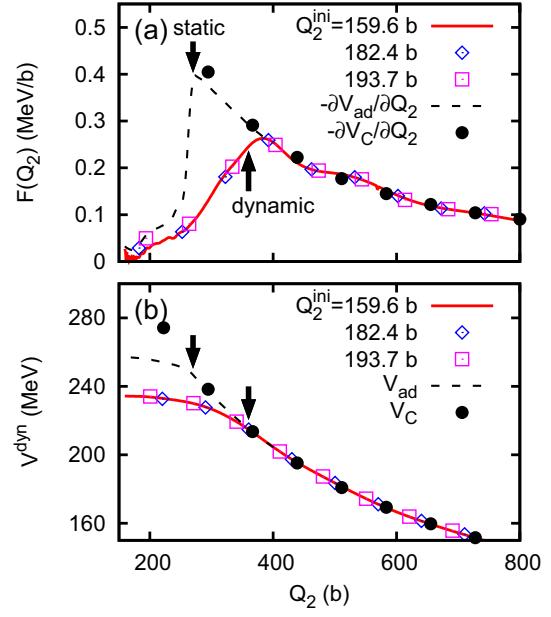

Figure 5. (Color online) (a) Function $F\left(Q_{2}\right)$ obtained with TDEDF using Eq. (18) for the three evolutions with $Q_{2}^{\text {ini }} \leq Q_{2}^{\text {sc }}$ displayed in Fig. 4. For comparison, we also show the forces acting on $Q_{2}$ that would be induced either by the adiabatic potential (black dashed line) or solely by the Coulomb field (black filled circle). The arrows in the figure indicate the $Q_{2}$ value where the neck density $\rho_{\text {neck }}$ becomes ten times less than the saturation density $\rho_{\text {sat }}=0.16 \mathrm{fm}^{-3}$. The two arrows indicate the adiabatic (static) and dynamical paths (dynamic). In the latter case, the position is almost independent of $Q_{2}^{\text {ini }}$. (b) Dynamical potential curve obtained by integrating $F\left(Q_{2}\right)$ using Eq. (22). Again for comparison, the adiabatic potential and the Coulomb field are also shown.

To access the potential and dissipative collective properties, it is convenient to define the quantity

$$
F\left(Q_{2}\right) \equiv \dot{P}_{2}-\frac{1}{2} \frac{\partial M_{2}}{\partial Q_{2}} \dot{Q}_{2}^{2}
$$

This function is shown in Fig. 5 for some of the evolutions presented in Fig. 4. If the macroscopic transport equation (17) is valid, this quantity is expected to identify with:

$$
F\left(Q_{2}\right)=-\frac{\partial V_{\text {coll }}}{\partial Q_{2}}-\gamma\left(Q_{2}\right) \dot{Q}_{2} .
$$

and therefore is sensitive to both the potential and dissipative part. For comparison, we also display the cases where dissipation is assumed to be zero in Eq. (19) and where the potential part identifies either to the adiabatic potential or solely to the Coulomb field. In the latter case, the Coulomb potential for large relative distance, or large $Q_{2}$, is approximated by

$$
V_{C} \approx \frac{Z_{1} Z_{2} e^{2}}{R} \approx \frac{Z_{1} Z_{2} e^{2}}{\sqrt{\frac{A}{2 A_{1} A_{2}} Q_{2}}}=\frac{1}{4} \sqrt{\frac{A}{2}} Z^{2} e^{2} Q_{2}^{-1 / 2} .
$$

The last expression is obtained for the symmetric fission case, i.e. $Z_{1}=Z_{2}=Z / 2$ and $A_{1}=A_{2}=A / 2$ considered here, and further assuming no intrinsic quadrupole deformation of emitted fragments after scission.

Figure 5(a) gives interesting information on the different steps leading to fission. We first see that after the very first instant of the evolution where some dissipation occurred, all evolutions obtained with $Q_{2}^{\text {ini }} \leq Q_{2}^{\text {sc }}$ are on top of each other. The dynamic before scission deviates significantly from the expected adiabatic one underlining the importance of both non-adiabatic and dissipative effects. In particular, we clearly see that the dynamical formation of the neck differs from the adiabatic case. Defining the scission point as the $Q_{2}$ value where the neck density equal $1 / 10$ of the saturation density, we observe that dynamically the scission occurs at much larger $Q_{2}$ than the adiabatic case (arrows in Fig. 5). This has two consequences (i) first, the two nuclei stick together up to a larger distance compared to the adiabatic case. Accordingly, the nuclear field can play an enhanced role. (ii) we see that we should introduce the notion of "dynamical scission point" that a priori differs from the "adiabatic scission point" and that occurs at larger quadrupole moment. In the present case of symmetric compact fission, the dynamical scission point occurs around $Q_{2}^{\text {scdyn }} \simeq 360 \mathrm{~b}$, compared to the adiabatic scission point $Q_{2}^{\text {sc,stat }} \simeq 270$ b.

After scission, the dynamical evolution is very close to the Coulomb field case (black filled circles). This indicates that no dissipation takes place after this point. Some oscillations observed around the average Coulomb repulsion are due to intrinsic oscillation of each fragment. These oscillations obviously go beyond the simple macroscopic approximation (17) since they involve additional intrinsic shape degrees of freedom.

Figure 4 seems to indicate that dissipation occurs only at rather small $Q_{2}$. For $Q_{2}>300 \mathrm{~b}$, one might assume that the motion is only driven by a potential that we denote by $V^{\mathrm{dyn}}\left(Q_{2}\right)$. Then, we have the approximate relationship:

$$
\frac{\partial V^{\mathrm{dyn}}\left(Q_{2}\right)}{\partial Q_{2}}=-F\left(Q_{2}\right)
$$

where $F\left(Q_{2}\right)$ is estimated along the path using Eq. (18). The potential may be obtained through the relation:

$$
V^{\mathrm{dyn}}\left(Q_{2}\right)=V_{C}\left(Q_{2}^{\max }\right)+\int_{Q_{2}}^{Q_{2}^{\max }} F\left(Q_{2}^{\prime}\right) d Q_{2}^{\prime}
$$

where the boundary $Q_{2}^{\max }$ is taken much larger than the dynamical scission point so that the potential at the boundary is dominated by the Coulomb repulsion between the fragments. Examples of potential obtained in this way is shown in Fig. 5(b) assuming $Q_{2}^{\max }=800$ b. $V_{\text {ad }}$ shown with the dashed curve in Fig. 5(b) is drawn by shifting the PEC given in Fig. 1 so that it coincides with $V_{C}$ at $Q_{2}=433 \mathrm{~b}\left(\geq Q_{2}^{\text {sc,stat }}\right)$.

We see in Fig. 5(b) that the potential obtained using Eq. (22) differs significantly from the adiabatic one at small $Q_{2}$ due to dynamics and eventually non-adiabatic effects. Note that the dynamical potential should be interpreted with some caution since it might contain some dissipative effects especially at initial time. It is worth in particular mentioning that the adiabatic and dynamical potentials should be identical at initial $Q_{2}$. We clearly observe in Fig. 5(b) a lower value for the dynamical case. The difference between the adiabatic and dynamical curves at $t=0$ corresponds to the energy transferred into the other collective degrees freedom or internal excitations of the fragments during the fission. We see that this difference is $\approx 23 \mathrm{MeV}$ for $Q_{2}^{\text {ini }}=160 \mathrm{~b}$. 


\section{Conclusion}

In the present work, a method is proposed to construct conjugated collective momenta associated to a given set of local collective variables along a TD-EDF path. A detailed discussion is made on the proper definition of associated inertia including the effect of its possible off-diagonal matrix elements. Once pairs of conjugated collective variables are obtained, one can make a macroscopic reduction of the microscopic mean-field dynamic.

An illustration is given here with the fission process. A precise analysis is made in the symmetric fission of ${ }^{258} \mathrm{Fm}$. The mass matrix is calculated along the fission path including only quadrupole moment, and/or both the quadrupole and hexadecapole moment. In particular, the important role of off-diagonal matrix elements of the mass is underlined. Then, a detailed analysis of the macroscopic evolution in the quadrupole collective space is made. The importance of dissipation in the early stage of the evolution is discussed. Clear non-adiabatic effects are probed, in particular associated with the specific neck evolution. We show that the dynamical scission occurs at much larger quadrupole moment compared to that for the adiabatic path. An attempt to extract the nucleus-nucleus potential felt by the daughter nuclei after fission is also made. It is shown that this potential significantly differs from the adiabatic one due to the non-adiabatic effects and the dissipation of energy into the intrinsic excitations of fission fragments.

It is worth mentioning that the method presented here is rather versatile and could be used in other dynamical processes. For instance, it could a priori be used to study anharmonicity in giant resonances as well as possible coupling between collective modes.

\section{Acknowledgements}

We would like to thank G. Adamian, N. Antonenko, S. Ayik and K. Hagino for useful discussions on collective observables. G.S. acknowledges the Japan Society for the Promotion of Science for the JSPS postdoctoral fellowship for foreign researchers. This work was supported by Grant-in-Aid for JSPS Fellows No. $14 \mathrm{~F} 04769$.

\section{References}

[1] K.-H. Kim, T. Otsuka, and P. Bonche, J. Phys. G 23, 1267 (1997)

[2] C. Simenel, Ph. Chomaz, and G. de France, Phys. Rev. Lett. 86, 2971 (2001)

[3] T. Nakatsukasa and K. Yabana, Phys. Rev. C 71, 024301 (2005)

[4] J. A. Maruhn, P.-G. Reinhard, P. D. Stevenson, J. R. Stone, and M. R. Strayer, Phys. Rev. C 71, 064328 (2005)
[5] A. S. Umar and V. E. Oberacker, Phys. Rev. C 71, 034314 (2005)

[6] K. Washiyama and D. Lacroix, Phys. Rev. C 78, 024610 (2008)

[7] B. Avez, C. Simenel, and Ph. Chomaz, Phys. Rev. C 78, 044318 (2008)

[8] S. Ebata, T. Nakatsukasa, T. Inakura, K. Yoshida, Y. Hashimoto, and K. Yabana, Phys. Rev. C 82, 034306 (2010)

[9] I. Stetcu, A. Bulgac, P. Magierski, and K. J. Roche, Phys. Rev. C 84, 051309 (2011)

[10] G. Scamps, D. Lacroix, G. F. Bertsch, and K. Washiyama, Phys. Rev. C 85, 034328 (2012)

[11] Y. Hashimoto, Eur. Phys. J. A 48, 1 (2012)

[12] G. Scamps and D. Lacroix, Phys. Rev. C 87, 014605 (2013)

[13] C. Simenel and A. S. Umar, Phys. Rev. C 89, 031601 (2014)

[14] P. M. Goddard, A Microscopic Study of Nuclear Fission using the Time-Dependent Hartree-Fock Method (PhD thesis, University of Surrey, 2014)

[15] P. M. Goddard, P. D. Stevenson, A. Rios, arXiv:1504.00919 (2015)

[16] G. Scamps, C. Simenel and D. Lacroix, Phys. Rev. C 92, 011602(R) (2015)

[17] R. Vandenbosch, J. R. Huizenga, Nuclear Fission (Academic Press, New York, 1973)

[18] J.W. Negele, Nucl. Phys. A 502, 371c (1989)

[19] H. Goutte, J. F. Berger, P. Casoli, and D. Gogny, Phys. Rev. C 71, 024316 (2005)

[20] C. Simenel, D. Lacroix, and B. Avez, Quantum Many-Body Dynamics: Applications to Nuclear Reactions (VDM Verlag, Sarrebruck, Germany, 2010)

[21] Y. Tanimura, D. Lacroix, and G. Scamps, Phys. Rev. C 92034601 (2015)

[22] P.-G. Reinhard, K. Goeke, and R. Y. Cusson, Z. Phys. A 295, 45 (1980)

[23] G. Bertsch and H. Feldmeier, Phys. Rev. C 56, 839 (1997)

[24] G.G. Adamian et al., Nucl. Phys. A 584, 205 (1995)

[25] P.-G. Reinhard, M. Brack and O. Genzken, Phys. Rev. A 41, 5568 (1990)

[26] P.-G. Reinhard, Y.-K. Gambhir, Annalen der Physik 504, 598 (1992)

[27] P. Bonche, H. Flocard, and P. H. Heenen, Comp. Phys. Com. 171, 49 (2005)

[28] G. Scamps and D. Lacroix, Phys. Rev. C 88, 044310 (2013)

[29] G. Scamps and D. Lacroix, Phys. Rev. C 89, 034314 (2014) 